\title{
Evaluation of Diagnostic Methods and Antimicrobial Susceptibility Pattern of Asymptomatic Bacteriuria Among Pregnant Women in Ashanti Region, Ghana
}

\author{
Desmond O. Acheampong ${ }^{1 *}$, Michael K. Afoakwah ${ }^{1}$, Alex Boye ${ }^{2}$, Richard Opoku ${ }^{1}$, \\ Godwin Kwakye-Nuako ${ }^{1}$, Christian K. Adokoh ${ }^{1}$, Samuel A. Baafi ${ }^{3}$ and Daniel Somuah ${ }^{4}$ \\ ${ }^{1}$ Department of Biomedical Sciences, University of Cape Coast, Cape Coast, Ghana; ${ }^{2}$ Department of Medical Laboratory Technology, \\ University of Cape Coast, Cape Coast, Ghana; ${ }^{3}$ Ellolab Diagnostic Centre, Kumasi, Ghana; ${ }^{4}$ Anglogold Ashanti Health Foundation \\ Hospital, Obuasi, Ghana
}

\begin{abstract}
Background and objective: Asymptomatic bacteriuria (ASB) poses a serious health problem to pregnant women and fetuses. However, in most developing countries, routine screening for ASB and antimicrobial sensitivity test are rarely performed. This study, therefore, aimed to determine the best diagnostic method for routine screening of ASB and antimicrobial susceptibility pattern among pregnant women attending an antenatal clinic in the Ashanti Region of Ghana.

Methods: Urine samples from 412 pregnant women between the ages of 16 and 45 years-old attending antenatal clinic at Anglogold Ashanti Health Foundation Hospital and Ellolab Diagnostic Centre were screened for ASB by microscopy, dipstick urinalysis and bacteria culture. Susceptibility of the positive isolates were assessed against commonly used antimicrobial agents, adopting the disc diffusion test method.
\end{abstract}

Results: Of the 412 pregnant women screened, 72 tested positive for ASB by the urine culture method, which translates into an overall prevalence of $17.5 \%$. There was no association between age, marital status, occupation, parity, educational background nor duration of pregnancy with ASB $(p>0.05)$. Additionally, dipstick urinalysis was found to be a better diagnostic method than microscopy. The most isolated bacteria were Escherichia coli (62.5\%) and Klebsiella pneumoniae (30.6\%), and nitrofurantoin and nalidixic acid were the most effective antimicrobial agents.

Conclusions: Routine urine culture and antimicrobial susceptibility test should be carried out on all pregnant women attending antenatal clinic to detect possible ASB and prescribe appropriate drugs, such as nitrofurantoin and nalidixic acid, to prevent any related complications. However, in health centers that lack bacterial culture facilities, dipstick urinalysis should be the preferred screening option.

Keywords: Asymptomatic; Bacteriuria; Antimicrobial agent; Diagnostic methods. Abbreviations: ASB, asymptomatic bacteriuria; UTI, urinary tract infection; PPV, positive predictive value; NPV, negative predictive value.

Received: January 19, 2018; Revised: April 13, 2018; Accepted: April 16, 2018

${ }^{*}$ Correspondence to: Desmond O. Acheampong, Department of Biomedical Sciences, School of Allied Health Sciences, University of Cape Coast, Cape Coast, Ghana Tel: +233 543710234, E-mail: dacheampong@ucc.edu.gh; do.acheampong@gmail. com

How to cite this article: Acheampong DO, Afoakwah MK, Boye A, Opoku R, Kwakye-Nuako G, Adokoh CK, Baafi SA, Somuah D. Evaluation of Diagnostic Methods and Antimicrobial Susceptibility Pattern of Asymptomatic Bacteriuria Among Pregnant Women in Ashanti Region, Ghana. J Explor Res Pharmacol 2018;3(3):78-84. doi: 10.14218/JERP.2018.00003.
Introduction

Pregnancy is commonly associated with urinary tract infection (UTI), and has been attributed to hormonal and physiological changes that occur during pregnancy. ${ }^{1}$ At week 6 of most pregnancies, the ureters begin to dilate as a result of the physiological changes, a condition commonly referred to as hydronephrosis of pregnancy. ${ }^{2}$ This condition peaks at 22-26 weeks and persists until delivery. ${ }^{2,3}$ Interestingly, both progesterone and estrogen levels increase during pregnancy and lead to decreases urethral and bladder tone. Increased bladder volume and decreased bladder tone along with decreased urethral tone contribute to increased urinary stasis 
and ureterovesical reflux, which could result in asymptomatic bacteriuria (ASB). Additionally, the uterus sits directly on top of the urinary bladder, and as the resultant weight of the uterus increases, it blocks the drainage of urine from the bladder, thus causing urinary stasis that might lead to infection of the urinary tract. ${ }^{1}$

Moreover, the immune system of pregnant women becomes compromised, rendering the pregnant woman more susceptible to both pathogenic and nonpathogenic microorganisms. Another important predisposing factor is the female sex itself, due to the relatively shorter urethra. Thus, proximity of the vagina to the anus, coupled with the inability of pregnant women to completely empty their bladder, predispose them to UTI. ${ }^{4}$ Finally, the anatomical link between the urethra and vagina makes it liable to trauma during sexual intercourse, lending further susceptibility to infections. ${ }^{5}$

Untreated ASB during pregnancy can adversely affect both mother and fetus, and is related to a greater chance of progression to pyelonephritis and possibly an increased susceptibility to preeclampsia, premature birth and low neonatal birth weight. ${ }^{6}$ It is, therefore, imperative to routinely screen pregnant women attending antenatal clinic for ASB, to be able to initiate remedial measures early enough to curb any related complications, should ASB be detected. The diagnostic methods commonly used for this purpose are the diagnostic dipstick, microscopy and urine culture; the latter being the most reliable. ${ }^{7}$ Urine culture is the gold standard against which the other two diagnostic techniques are evaluated. ${ }^{8}$

Gram-negative bacteria, such as Escherichia coli, Klebsiella and Proteus, and Gram-positive bacteria, such as hemolytic Streptococci and Staphylococcus saprophyticus, are the most implicated bacteria species for ASB in pregnancy. ${ }^{9}$ Earlier studies conducted in Ghana found UTI prevalence of $7.3 \%,{ }^{10,11}$ with E. coli being the dominant bacteria isolated from the pregnant women who attended antenatal care during the study period. These high prevalence rates in the earlier studies paint a gloomy picture that requires immediate and appropriate interventions. ${ }^{10,11}$ Unlike the bacteria culture method, the dipstick and microscopy diagnostic methods readily produce results in a short space of time, which is an important requirement in the routine diagnostic method. Therefore, any of these two diagnostic methods that proves efficient can be recommended to be used to screen pregnant women for ASB.

The use of antibiotics is, to a greater extent, the only important modifiable risk factor for antibiotic resistance. ${ }^{12}$ Most often, treatment of UTI is started empirically and it is based on the prior information physicians have on the antimicrobial resistance patterns of the urinary pathogens. ${ }^{13}$ However, in recent times many reports have been published on increased antimicrobial resistance among urinary tract isolates, from all over the globe. ${ }^{14,15}$ In African countries, including Ghana, the use of drugs that are not up to standard, indiscriminate use of antibiotics and erratic prescription by unqualified drug peddlers have been identified as contributing to the surge in antimicrobial resistance. ${ }^{16}$

The current study aimed to assess the effectiveness of microscopy and dipstick diagnostic methods for diagnosing ASB compared to the bacteria culture method (as gold standard), estimating the prevalence of ASB and investigating the antimicrobial susceptibility pattern of the bacteria responsible for ASB among pregnant women attending antenatal care in the Ashanti region, Ghana.

\section{Methods}

\section{Study site}

This study was undertaken from January 2017 to May 2017 at the Anglogold Ashanti Health Foundation Hospital in Obuasi and Ellolab Diagnostic Centre in Kumasi, both located in the Ashanti region of Ghana.

\section{Questionnaire administration}

Questionnaires were administered to pregnant women attending antenatal clinic at these hospitals during the study period. However, pregnant women with clinical signs and symptoms of UTI and those who were on antimicrobial treatment prior to sample collection were excluded. Respondents were briefed about the study and their consent sought before administering the questionnaires. A total of 412 questionnaires were administered to access certain vital sociodemographic data from the study subjects. Each interview lasted for 5 to $10 \mathrm{~m}$.

\section{Collection and processing of urine samples}

The women were educated about the correct procedures for collecting a urine sample for clinical purposes. Subsequently, sterile urine containers were given to them to collect a clean-catch midstream urine sample for the test. Urine samples were preliminarily analyzed for ASB by Multistix 10SG urine test strips (Siemens Healthcare Diagnostics, Tarrytown, NY, USA). This was done within $2 \mathrm{~h}$ of sample collection. A Multistix 10SG urine strip (Siemens Healthcare Diagnostics) was dipped in the urine sample and analyzed according to the manufacturer's instructions. Subsequently, microscopic analysis was performed (Model CX22; Olympus, Tokyo, Japan). The urine samples were cultured within $2 \mathrm{~h}$ of sample collection. Using a calibrated wire inoculating loop $(0.001 \mathrm{~mL})$, urine samples were inoculated into cystine lactose electrolyte-deficient medium (Liofichem, Ltd., Piane Vomano, Italy) and incubated at $37^{\circ} \mathrm{C}$ for $24 \mathrm{~h}$. Colonies were counted to check the presence of significant bacteriuria.

Colony count yielding bacterial growth of $10^{5} \mathrm{CFU} / \mathrm{mL}$ of urine was regarded as significant bacteriuria. Subsequent to this, the specificity, sensitivity, positive predictive value (PPV) and negative predictive value (NPV) of microscopy and dipstick diagnostic methods were calculated using the following formulae: sensitivity $=$ number of true positives $\div$ (number of true positives + number of false negatives); specificity $=$ number of true negative $\div$ (number of true negatives + number of false positives); PPV $=$ number of true positive $\div$ (number of true positives + number of false positives); NPV $=$ number of true negative $\div$ (number of true negative + number of false negatives).

Bacterial isolates were then identified based on their colonial morphology, the Gram reaction and pattern of biochemical reactions. The disc diffusion antimicrobial sensitivity test was subsequently performed for the positive cultures using antimicrobial sensitivity discs on Mueller-Hinton agar (Oxoid Ltd., Hampshire, UK) as instructed by the standardized table published by the Clinical and Laboratory Standards Institute (2014). Antimicrobial discs tested against the positive isolates included ampicillin $(10 \mu \mathrm{g})$, cefuroxime $(30 \mu \mathrm{g})$, cotrimoxazole $(25 \mu \mathrm{g})$, gentamicin $(10 \mu \mathrm{g})$, tetracycline (30 $\mu \mathrm{g})$, nalidixic acid $(30 \mu \mathrm{g})$, nitrofurantoin $(300 \mu \mathrm{g})$, pipemidic acid $(20 \mu \mathrm{g})$ and meropenem $(10 \mu \mathrm{g})$ (Oxoid Ltd.). Reference strains of E. coli (No. 25922; American Type Culture Collection, Manassas, VA, USA) and S. aureus (No. 25923; American Type Culture Collection) preserved in our laboratory were used as controls.

The protocol was approved and ethically cleared by the Department of Biomedical Sciences, College of Health and Allied Sciences, University of Cape Coast (Ghana), and written informed consent was obtained from all patients prior to enrollment. Ethical approval was also obtained from the Anglogold Ashanti Health 
Table 1. Prevalence of asymptomatic bacteriuria based on sociodemographic characteristics determined by the culture method

\begin{tabular}{|c|c|c|c|c|c|}
\hline Characteristic & Total Number, $n(\%)$ & Tested Negative, $\boldsymbol{n}(\%)$ & Tested Positive, $n$ (\%) & Chi-Square Value, $X^{2}$ & $p$-value \\
\hline \multicolumn{6}{|l|}{ Age } \\
\hline $16-20$ & $26(6.3)$ & $23(88.5)$ & $3(11.5)$ & 3.146 & 0.678 \\
\hline $21-25$ & $86(20.9)$ & $74(86.0)$ & $12(14.0)$ & & \\
\hline $26-30$ & $154(37.4)$ & $125(81.2)$ & $29(18.8)$ & & \\
\hline $31-35$ & 77 (18.7) & $64(83.1)$ & $13(16.9)$ & & \\
\hline $36-40$ & 47 (11.4) & 38 (80.9) & 9 (19.1) & & \\
\hline $41-45$ & $22(5.3)$ & $16(72.7)$ & $6(27.3)$ & & \\
\hline \multicolumn{6}{|l|}{ Marital status } \\
\hline Single & 73 (17.7) & $58(79.5)$ & $15(20.5)$ & 0.581 & 0.272 \\
\hline Married & 339 (82.3) & $282(83.2)$ & $57(16.8)$ & & \\
\hline \multicolumn{6}{|l|}{ Educational background } \\
\hline Illiterate & $22(5.3)$ & 20 (90.9) & $2(9.1)$ & 11.651 & 0.109 \\
\hline Primary & $30(7.3)$ & $26(86.7)$ & $4(13.3)$ & & \\
\hline Secondary & $229(55.6)$ & 198 (86.5) & 31 (13.5) & & \\
\hline Higher learning & $131(31.8)$ & $96(73.3)$ & $35(26.7)$ & & \\
\hline \multicolumn{6}{|l|}{ Occupation } \\
\hline Student & $13(3.2)$ & $11(84.6)$ & $2(15.4)$ & 3.959 & 0.138 \\
\hline Housewife & $66(16.0)$ & $60(90.9)$ & $6(9.1)$ & & \\
\hline Employed & $333(80.8)$ & $269(80.8)$ & $64(19.2)$ & & \\
\hline \multicolumn{6}{|l|}{ Parity } \\
\hline$<1$ & 149 (36.2) & 119 (79.9) & $30(20.1)$ & 1.636 & 0.802 \\
\hline 1 & $103(25.0)$ & $86(83.5)$ & $217(23.6)$ & & \\
\hline 2 & $68(16.5)$ & $56(82.4)$ & $12(17.6)$ & & \\
\hline 3 & $54(13.1)$ & $47(87.0)$ & $7(13.0)$ & & \\
\hline$\geq 4$ & $38(9.2)$ & $32(84.2)$ & $6(15.8)$ & & \\
\hline \multicolumn{6}{|l|}{ Duration of pregnancy } \\
\hline First trimester & $34(8.3)$ & $31(91.2)$ & $3(8.8)$ & 2.891 & 0.236 \\
\hline Second trimester & $117(28.4)$ & $99(84.6)$ & $18(15.4)$ & & \\
\hline Third trimester & $261(63.3)$ & $210(80.5)$ & $51(19.5)$ & & \\
\hline
\end{tabular}

Foundation Hospital and Ellolab Diagnostic Center. All procedures were performed in accordance with the ethical standards outlined in the Helsinki Declaration of 1975, as revised in 2013.

\section{Statistical analysis}

Data was analyzed using the SPSS software package version 21 (IBM Corp., Armonk, NY, USA). The data were analyzed using chisquare $\left(\chi^{2}\right)$ test, to test whether differences between values are significant. $p$-values less than 0.05 were considered statistically significant.

\section{Results}

The ages of the study subjects ranged from 16 to 45 years, with the majority ( $n=154,37.4 \%$ ) belonging to the age group of $26-30$ years (Table 1). The mean and median ages were 29.27 and 29 years respectively, whereas the modal age was 28 years. Educational status of participants included illiterate $(n=22,5.3 \%)$, primary school level $(n=30,7.3 \%)$, secondary school level $(n=229$, $55.6 \%)$ and tertiary level $(n=131,31.8 \%)$. Among the 412 study participants, $34(8.3 \%), 117(28.4 \%)$ and $261(63.3 \%)$ were in the first, second and third trimesters respectively (Table 1 ). A total of $73(17.7 \%)$ of the women were single (unmarried), whereas the remaining $339(82.3 \%)$ were married (Table 1$)$.

Of the 412 pregnant women, 72 were found to be positive for ASB by the urine culture method (Table 2), which translates into an overall prevalence of $17.5 \%$. The age group of $41-45$ years presented the highest prevalence, being $27.3 \%$ (Table 1). Also, as shown in Table 1, the pregnant women in the third trimester showed the highest prevalence, being $19.5 \%$. Prevalence of ASB 
Table 2. Overall prevalence of symptomatic bacteriuria based on microbial culture and microscopy

\begin{tabular}{lll}
\hline & Negative, $\boldsymbol{n}$ (\%) & Positive, $\boldsymbol{n}$ (\%) \\
\hline Microbial culture & $340(82.5)$ & $72(17.5)$ \\
Microscopy & $341(82.8)$ & $71(17.2)$ \\
Dipstick & $338(82.0)$ & $74(18.0)$ \\
\hline
\end{tabular}

among pregnant women based on microscopy was $17.2 \%$ (Table 2). Also, presented in Table 3 is prevalence of symptomatic bacteriuria using various dipstick parameters, and prevalence of ASB based on dipstick diagnostic method was $18.0 \%$ (Table 2). The sensitivity, specificity, PPV and NPV of microscopy as calculated from Table 4 were $74.32 \%, 94.9 \%, 76.3 \%$ and $94.4 \%$ respectively. The sensitivity, specificity, PPV and NPV of dipstick urinalysis as calculated from Table 4 were $95.9 \%, 99.7 \%, 98.6 \%$ and $99.1 \%$ respectively. These were determined based on leukocyte esterase and nitrite parameters.

Among the isolated bacteria species ( $n=72,17.5 \%)$, the Gramnegative bacteria E. coli, K. pneumoniae and P. mirabilis were the most prevalent $(n=71,98.6 \%)$ compared to the Gram-positive Enterococcus fecalis $(n=1,1.4 \%$; Table 5$)$. The most dominant bacteria isolated was $E$. coli $(n=45,62.5 \%)$, followed by K. pneumoniae $(n=22,30.6 \%)$. As shown in Table 6, E. coli was relatively sensitive to nitrofurantoin (75.6\%) and nalidixic Acid (57.8). K. pneumoniae also demonstrated a similar trend to nalidixic acid $(72.7 \%)$ and nitrofurantoin $(63.6 \%)$. P. mirabilis, on the other hand, exhibited $50 \%$ sensitivity to both nalidixic acid and meropenem. E. fecalis, the only Gram-positive strain, was sensitive to only meropenem $(100 \%)$.

\section{Discussion}

Pregnant women at any stage of pregnancy are at increased risk of UTI, although such infections are usually asymptomatic. ${ }^{17,18}$ In this study of the prevalence of ASB in pregnant women, suitable routine screening methods and antimicrobial susceptibility patterns of the isolates were investigated. In the quest to recommend an efficient routine diagnostic method that could produce results in relatively shorter time for possible ASB, the sensitivity and specificity of the microscopic and dipstick diagnostic methods were also determined and compared. The diagnosis of ASB in this study was based on a single urine sample from each study participant.

Based on bacterial culture method, the gold standard diagnostic method, the overall prevalence of ASB among pregnant women in this study was $17.5 \%$. This prevalence was relatively higher than those reported in earlier studies, including those from Ethiopia $(3.3 \%$ and $11.6 \%),{ }^{19,20} \operatorname{Iran}(6.1 \%),{ }^{21}$ Ghana $(7.3 \%)$ and Mexico $(8.4 \%){ }^{10,22}$ It was however lower than in other studies carried out in Nigeria (22.2\%) and Ghana (56.5\%). ${ }^{11,23}$ It can, therefore, be

Table 3. Prevalence of symptomatic bacteriuria using various dipstick parameters

\begin{tabular}{lll}
\hline & Negative, $\boldsymbol{n}(\%)$ & Positive, $\boldsymbol{n}(\%)$ \\
\hline Leukocytes & $352(85.4)$ & $60(14.6)$ \\
Nitrites & $341(82.8)$ & $71(17.2)$ \\
Proteins & $400(97.1)$ & $12(2.9)$ \\
Blood & $404(98.1)$ & $8(1.9)$ \\
\hline
\end{tabular}

Table 4. Comparison between dipstick urinalysis and microscopy using bacteria culture as standard

\begin{tabular}{|c|c|c|c|c|c|}
\hline & & \multicolumn{2}{|c|}{ Dipstick } & \multicolumn{2}{|c|}{ Microscopy } \\
\hline & & Positive & Negative & Positive & Negative \\
\hline \multirow{3}{*}{ Culture } & Positive & 71 & 1 & 54 & 18 \\
\hline & \multirow{2}{*}{ Negative } & 3 & 337 & 17 & 323 \\
\hline & & 74 & 338 & 71 & 341 \\
\hline
\end{tabular}

inferred from the above results that the prevalence of ASB varies from one geographical location to another, which can possibly be attributed to differences in the mode of screening and/or compounding risk factors such as age, parity, educational level, environmental and personal hygiene. ${ }^{11,24}$ Notwithstanding, the $17.5 \%$ prevalence recorded in this study is relatively high, and considering the demography of study locations, it could possibly represent the national prevalence of Ghana. It, therefore, calls for concerted efforts among all interested parties to formulate a national policy to help curb this situation.

The current study did not find any association between age, marital status, occupation, parity, educational background and duration of pregnancy $(p>0.05)$ for positive results of ASB, and implies that the incidence of ASB among pregnant women might not be dependent on these demographic parameters and therefore might not be considered risk factors. This was consistent with similar studies conducted in Ethiopia. ${ }^{25,26}$ The highest prevalence of this study was recorded for the age group of 41-45 years and the lowest for the age group of 16-20 years. The finding of the highest and lowest prevalence rates being represented by the oldest and youngest age groups, respectively, was very instructive. This relatively high prevalence among the older women might be due to a compromised immune system, and also, during pregnancy, the lining of the tissues around the vagina becomes more fragile, which get worse with age, making older pregnant women more susceptible and younger ones less susceptible to the infections. ${ }^{27}$

This study also recorded high ASB prevalence among pregnant women in the third trimester, which was followed by the second trimester of pregnancy. This was consistent with the outcome of earlier studies in Nigeria. ${ }^{28,29}$ Possibly, susceptibility to ASB at this stage of pregnancy could be due to dilatation of the ureters, which starts around 6 weeks and reaches the peak at weeks $22-24 .{ }^{30}$ It could also be due to poor personal hygiene since most pregnant women at this stage find it difficult to clean thoroughly. This was, however, inconsistent with the findings of Obirikorang et al. ${ }^{24}$ which indicated highest prevalence occurring in the second trimester, whereas Boye et al. ${ }^{11}$ and Turpin et al. ${ }^{10}$ recorded highest prevalence in the first trimester in Ghana.

This study showed that the dipstick diagnostic test has higher sensitivity and specificity than the urine microscopy test, indicating that dipstick urinalysis was a better diagnostic method for ASB

Table 5. Frequency of isolated bacteria species

\begin{tabular}{ll}
\hline Bacterial Species & Frequency, $\boldsymbol{n}(\%)$ \\
\hline E. coli & $45(62.5)$ \\
Klebsiella pneumoniae & $22(30.6)$ \\
Enterococcus fecalis & $1(1.4)$ \\
Proteus mirabilis & $4(5.6)$ \\
Total & 72 \\
\hline
\end{tabular}


Table 6. Susceptibility pattern of isolates to commonly used antimicrobial agents

\begin{tabular}{|c|c|c|c|c|c|}
\hline Antibiotic & Sensitivity & E. coli, $n(\%)$ & Klebsiella pneumoniae, $n(\%)$ & Proteus mirabilis, $n$ (\%) & Streptococcus fecalis, $n$ (\%) \\
\hline \multirow[t]{2}{*}{ Ampicillin } & Resistant & $45(100.0)$ & $22(100.0)$ & $4(100.0)$ & $1(100.0)$ \\
\hline & Sensitive & $0(0.0)$ & $0(0.0)$ & $0(0.0)$ & $0(0.0)$ \\
\hline \multirow[t]{2}{*}{ Cefuroxime } & Resistant & 45 (100.0) & $22(100.0)$ & $4(100.0)$ & $1(100.0)$ \\
\hline & Sensitive & $0(0.0)$ & $0(0.0)$ & $0(0.0)$ & $0(0.0)$ \\
\hline \multirow[t]{2}{*}{ Cotrimoxazole } & Resistant & $45(100.0)$ & $22(100.0)$ & $3(75.0)$ & $1(100.0)$ \\
\hline & Sensitive & $0(0.0)$ & $0(0.0)$ & $1(25.0)$ & $0(0.0)$ \\
\hline \multirow[t]{2}{*}{ Gentamicin } & Resistant & $38(84.4)$ & $18(81.8)$ & $3(75.0)$ & $1(100.0)$ \\
\hline & Sensitive & $7(15.6)$ & $4(18.2)$ & $1(25.0)$ & $0(0.0)$ \\
\hline \multirow[t]{2}{*}{ Tetracyclin } & Resistant & $45(100.0)$ & $22(100.0)$ & $4(100.0)$ & $1(100.0)$ \\
\hline & Sensitive & $0(0.0)$ & $0(0.0)$ & $0(0.0)$ & $0(0.0)$ \\
\hline \multirow[t]{2}{*}{ Nalidixic acid } & Resistant & $19(42.2)$ & $6(27.3)$ & $2(50.0)$ & $1(100.0)$ \\
\hline & Sensitive & $26(57.8)$ & $16(72.7)$ & $2(50.0)$ & $0(0.0)$ \\
\hline \multirow[t]{2}{*}{ Nitrofurantoin } & Resistant & $11(24.4)$ & $8(36.4)$ & $4(100.0)$ & $1(100.0)$ \\
\hline & Sensitive & 34 (75.6) & $14(63.6)$ & $0(0.0)$ & $0(0.0)$ \\
\hline \multirow[t]{2}{*}{ Pipemidic acid } & Resistant & $44(97.8)$ & $19(86.4)$ & $4(100.0)$ & $1(100.0)$ \\
\hline & Sensitive & $1(2.2)$ & $3(13.6)$ & $0(0.0)$ & $0(0.0)$ \\
\hline \multirow[t]{2}{*}{ Meropenem } & Resistant & $22(57.9)$ & $9(50.0)$ & $2(50.0)$ & $0(0.0)$ \\
\hline & Sensitive & $16(42.1)$ & $9(50.0)$ & $2(50.0)$ & $1(100.0)$ \\
\hline
\end{tabular}

than microscopy. This finding was consistent with an earlier study by Glissmeyer et al. ${ }^{31}$ Among the five parameters considered under dipstick analysis, nitrite was the most sensitive and, therefore, could be the appropriate parameter for the dipstick diagnostic method. The nitrite test precisely predicted 71 out of the 72 positive cases, where all of the 71 cases represented enterobacteriaceae colonization. The nitrite test is based on the ability of the bacteria to reduce nitrate to nitrite in the bladder urine. ${ }^{32}$ Reduction of nitrate to nitrite by bacteria is dependent on time, and a positive result requires a prolonged bladder incubation period of more than $4 \mathrm{~h}$ for significant residual urine.$^{33}$ The nitrite test was unable to predict the case of the Enterococcus colonization because the organism cannot reduce nitrate to nitrite, thereby accounting for the negative result. ${ }^{34}$ Both dipstick and microscopy had high NPV and PPVs in screening for possible ASB in pregnant women. However, the dipstick method had a higher sensitivity, specificity, PPV and NPV than microscopy. As such, the dipstick diagnostic method is apparently a better diagnostic method than microscopy. However, carrying out a urine culture as a confirmatory test is imperative for appropriate diagnosis.

This study has also demonstrated that $E$. coli is the dominant bacteria responsible for ASB in pregnant women, accounting for $62.5 \%$ of the ASB cases. This was corroborated by earlier studies by Boye et al. ${ }^{11}$ and Turpin et al. ${ }^{10}$ in Ghana. The high prevalence of $E$. coli might be due to a number of virulence factors specific for colonization and invasion of the urinary epithelium, such as the P-fimbria and S-fimbria adhesions associated with E. coli. ${ }^{35}$ Additionally, this could be due to urinary stasis common in pregnancy, because most $E$. coli strains thrive well in this environment and, therefore, are capable of producing ASB. ${ }^{36}$ The second dominant bacteria $K$. pneumoniae accounted for $30.6 \%$ of the ASB cases. This was also consistent with the findings by Boye et al. ${ }^{11}$ These bacteria are, therefore, the major cause of ASB in pregnant women.
E. coli the most commonly isolated bacteria in this study and was highly susceptible to nitrofurantoin and nalidixic acid but demonstrated slight sensitivity to meropenem and a relatively low sensitivity to gentamicin and pipemidic acid. It was, however, resistant to ampicillin, cefuroxime, tetracycline and cotrimoxazole; as such, these drugs would not be suitable for empirical treatment of ASB in these pregnant women. K. pneumoniae on the other hand was highly sensitive to nalidixic acid and nitrofurantoin. It also demonstrated $50 \%$ sensitivity to meropenem and was slightly sensitive to gentamicin and pipemidic acid. However, just as $E$. coli, it demonstrated resistance to ampicillin, cefuroxime, tetracycline and cotrimoxazole. E. fecalis was sensitive to meropenem only. P. mirabilis demonstrated $50 \%$ susceptibility to nalidixic acid and meropenem, $25 \%$ sensitivity to cotrimoxazole and gentamicin, and was also resistant to ampicillin, cefuroxime, tetracycline, nitrofurantoin and pipemidic acid.

Evidently, the level of resistance against antimicrobial agents has increased over time. ${ }^{37,38}$ This corroborates earlier reports about the increasing resistance to potent antimicrobials by bacteria responsible for UTI and can be attributed many factors, ${ }^{14,39}$ which include improper use of antimicrobial agents. In view of this development, antimicrobial sensitivity test is strongly recommended before a drug is prescribed to control this unpleasant occurrence. In situations where empirical treatment is unavoidable, potent antimicrobial agents, such as nitrofurantoin and nalidixic acid, should be considered. This study, therefore, supports the use of nitrofurantoin and nalidixic acid for empirical treatment of ASB in pregnant women. Nitrofurantoin works by destroying the DNA of bacteria during a highly reactive state at its reduced form. Nitrofurantoin is reduced rapidly by flavoproteins found in the bacterial cell to many reactive intermediates that react with DNA, ribosomal proteins and other important macromolecules within the cells. ${ }^{40}$ Nalidixic acid is a synthetic quinolone that works by inhibiting replication of 
DNA and transcription in the bacteria. ${ }^{41}$

\section{Future research directions}

The authors have an ongoing study that seeks to detect and characterize genes responsible for extended-spectrum beta-lactamases in extended-spectrum beta-lactamases-producing Gram-negative bacteria implicated for ASB in pregnant women in Ghana. Extended-spectrum beta-lactamases are plasmid-encoded enzymes implicated in resistance against beta-lactam antimicrobials, such as third-generation cephalosporins, and are associated with multipledrug resistance. ${ }^{42}$ The plasmids encoding the extended-spectrum beta-lactamases confer the antimicrobial resistance characteristic of the bacteria that harbors them. There is, therefore, the need for these studies because the bacteria isolates found in this study, which were mostly Gram-negative and belonging to the family Enterobacteriaceae, demonstrated high resistance to most of the commonly used antimicrobial agents. This follow-up study could provide the molecular basis for the ever-increasing resistance of bacteria against the hitherto potent antimicrobial agents.

\section{Conclusions}

In the study presented herein, the prevalence of ASB among pregnant women attending antenatal clinic was relatively high. It is, therefore, imperative to screen every pregnant woman that attends antenatal clinic in Ghana and other developing counties for ASB. This will help reduce the possible complications. The suitable diagnostic method for screening ASB should have high specificity and sensitivity, and produce the required results in a short space of time. The dipstick diagnostic method has proved to be more efficient than microscopy and, thus, recommended to be used for the screening of ASB among pregnant women in health centers with no facility for bacterial culture. Also, most of the isolates were relatively susceptible to nitrofurantoin and nalidixic acid and, therefore, are considered appropriate for empirical treatment of pregnant women diagnosed with ASB.

\section{Acknowledgments}

We appreciate the valuable contribution of the management and staff of Anglogold Ashanti Health Foundation Hospital and Ellolab Diagnostic Center to this study.

\section{Conflict of interest}

The authors have no conflict of interest related to this publication.

\section{Author contributions}

This study was carried out in collaboration between all authors. Authors DOA, MKF and RO designed the study, performed the statistical analysis, wrote the protocol, and wrote the first draft of the manuscript. Authors AB, SAB and DS managed the analyses of the study and edited the final manuscript for intellectual content. Authors CKA and GKN managed the literature searches. All authors read and approved the final manuscript.
References

[1] Mordi RM, Burke ME, Odjadjare EE, Enabulele SA, Umeh OJ. Prevalence of urinary tract infections (UTI) among pregnant women in University of Benin Teaching Hospital (UBTH) Benin City, Nigeria. J Asian Sci Res 2015;5(4):198-204. doi:10.18488/journal.2/2015.5.4/2.4.198.204.

[2] Finn M. Abbott J, Bowyer L, Finn M. The physiological changes of pregnancy. In: Abbott J, Bowyer L, Finn M, editors. Obstetrics and Gynaecology: an evidence-based guide. 2nd ed. Australia: Elsevier; 2014, p. 94-110.

[3] Tan EK, Tan EL. Alterations in physiology and anatomy during pregnancy. Best Pract Res Clin Obstet Gynaecol 2013;27(6):791-802. doi:10.1016/j.bpobgyn.2013.08.001.

[4] Sultan AH, Monga A, Lee J, Emmanuel A, Norton C, Santoro G, et al. An International Urogynecological Association (IUGA)/International Continence Society (ICS) joint report on the terminology for female anorectal dysfunction. Neurourol Urodyn 2017;36(1):10-34. doi:10.1002/nau.23055.

[5] Nwosu D, Ifeanyi OE, Amajioyi O, Ibebuike J, Ozims S. Prevalence of bacterial and parasitic urinary tract infections in female students of IMO state university. World J Pharm Pharm Sci 2015;4(5):152-167.

[6] Schnarr J, Smaill F. Asymptomatic bacteriuria and syptomatic urinary tract infection in pregnancy. Eur J Clin Invest 2008;38(Suppl 2):50-57. doi:10.1111/j.1365-2362.2008.02009.x.

[7] Birnie K, Hay AD, Wootton M, Howe R, MacGowan A, Whiting P, et al. Comparison of microbiological diagnosis of urinary tract infection in young children by routine health service laboratories and a research laboratory: Diagnostic cohort study. PloS one 2017;12(2):e0171113. doi:10.1371/journal.pone.0171113.

[8] Demilie T, Beyene G, Melaku S, Tsegaye W. Diagnostic accuracy of rapid urine dipstick test to predict urinary tract infection among pregnant women in Felege Hiwot Referral Hospital, Bahir Dar, North West Ethiopia. BMC Res Notes 2014;7:481. doi:10.1186/1756-05007-481.

[9] Szweda H, Jóźwik M. Urinary tract infections during pregnancy-an updated overview. Dev Period Med 2016;20(4):263-272.

[10] Turpin C, Minkah B, Danso K, Frimpong E. Asymptomatic bacteriuria in pregnant women attending antenatal clinic at komfo anokye teaching hospital, kumasi, ghana. Ghana Med J 2007;41(1):26-29.

[11] Boye A, Siakwa PM, Boampong JN, Koffuor GA, Ephraim RKD, Amoateng $\mathrm{P}$, et al. Asymptomatic urinary tract infections in pregnant women attending antenatal clinic in Cape Coast, Ghana. E3 J Med Res 2012;1(6):74-83.

[12] Costelloe C, Metcalfe C, Lovering A, Mant D, Hay AD. Effect of antibiotic prescribing in primary care on antimicrobial resistance in individual patients: systematic review and meta-analysis. BMJ 2010;340:c2096. doi:10.1136/bmj.c2096.

[13] Wilson ML, Gaido L. Laboratory diagnosis of urinary tract infections in adult patients. Clin Infect Dis 2004;38(8):1150-1158. doi:10.1086/383029.

[14] Zorc JJ, Kiddoo DA, Shaw KN. Diagnosis and management of pediatric urinary tract infections. Clin Microbiol Rev 2005;18(2):417-422. doi:10.1128/CMR.18.2.417-422.2005.

[15] Acheampong DO, Boamponsem LK, Feglo PK. Occurrence and species distribution of Klebsiella isolates: A case study at Komfo Anokye Teaching Hospital (KATH) in Ghana. Adv Appl Sci Res 2011;2(4):187193.

[16] Chinedum IE. Microbial resistance to antibiotics. Afr J Biotechnol 2005;4(13):1606-1611.

[17] Schneeberger C, Kazemier BM, Geerlings SE. Asymptomatic bacteriuria and urinary tract infections in special patient groups: women with diabetes mellitus and pregnant women. Curr Opin Infect Dis 2014;27(1):108-114. doi:10.1097/QCO.0000000000000028.

[18] Angelescu K, Nussbaumer-Streit B, Sieben W, Scheibler F, Gartlehner $\mathrm{G}$. Benefits and harms of screening for and treatment of asymptomatic bacteriuria in pregnancy: a systematic review. BMC Pregnancy Childbirth 2016;16(1):336. doi:10.1186/s12884-016-1128-0.

[19] Demilie T, Beyene G, Melaku S, Tsegaye W. Urinary bacterial profile and antibiotic susceptibility pattern among pregnant women in 
J Explor Res Pharmacol

North West Ethiopia. Ethiop J Health Sci 2012;22(2):121-128.

[20] Tadesse A, Negash M, Ketema L. Asymtomatic bacteriuria in pregnancy: assesment of prevlence, microbial agents and ther antimicrobial sensitivty pattern in Gondar Teaching Hospital, North West Ethiopia. Ethiop Med J 2007;45(2):143-149.

[21] Lavigne J-P, Boutet-Dubois A, Laouini D, Combescure C, Bouziges N, Marès $\mathrm{P}$, et al. Virulence potential of Escherichia coli strains causing asymptomatic bacteriuria during pregnancy. J Clin Microbiol 2011;49(11):3950-3953. doi:10.1128/JCM.00892-11.

[22] Hernández BF, López CJ, Rodríguez MJ, Peralta PM, Rodríguez GR, Ortiz AA. Asymptomatic bacteruiria frequency in pregnant women and uropathogen in vitro antimicrobial sensitivity. Ginecol Obstet Mex 2007;75(6):325-331.

[23] Olusanya O, Ogunledun A, Fakoya T. Asymptomatic significant bacteriuria among pregnant and non-pregnant women in Sagamu, Nigeria. West Afr J Med 1993;12(1):27-33.

[24] Obirikorang C, Quaye L, Bio F, Amidu N, Acheampong I, Addo K. Asymptomatic bacteriuria among pregnant women attending antenatal clinic at the university hospital, Kumasi, Ghana. J Med Biomed Sci 2012;1(1):38-44.

[25] Alemu A, Moges F, Shiferaw Y, Tafess K, Kassu A, Anagaw B, et al. Bacterial profile and drug susceptibility pattern of urinary tract infection in pregnant women at University of Gondar Teaching Hospital, Northwest Ethiopia. BMC Res Notes 2012;5:197. doi:10.1186/17560500-5-197.

[26] Hamdan HZ, Ziad AHM, Ali SK, Adam I. Epidemiology of urinary tract infections and antibiotics sensitivity among pregnant women at Khartoum North Hospital. Ann Clin Microbiol Antimicrob 2011;10:2. doi:10.1186/1476-0711-10-2.

[27] Ramjee G, Daniels B. Women and HIV in sub-Saharan Africa. AIDS Res Ther 2013;10(1):30. doi:10.1186/1742-6405-10-30.

[28] Onu FA, Ajah LO, Ezeonu PO, Umeora OUJ, Ibekwe PC, Ajah MI. Profile and microbiological isolates of asymptomatic bacteriuria among pregnant women in Abakaliki, Nigeria. Infect Drug Resist 2015;8:231235. doi:10.2147/IDR.S87052.

[29] Samet JM, Zeger SL, Dominici F, Curriero F, Coursac I, Dockery DW, et al. The national morbidity, mortality, and air pollution study. Part II: morbidity and mortality from air pollution in the United States. Res Rep Health Eff Inst 2000;94(Pt 2):5-70, discussion 71-79.

[30] Cox S, Reid F. Urogynaecological complications in pregnancy: an overview. Obstetrics, Gynaecology \& Reproductive Medicine 2015;25(5):123-127. doi:10.1016/j.ogrm.2015.02.002.
Acheampong DO. et al: Asymptomatic bacteriuria in pregnant women

[31] Glissmeyer EW, Korgenski EK, Wilkes J, Schunk JE, Sheng X, Blaschke AJ, et al. Dipstick screening for urinary tract infection in febrile infants. Pediatrics 2014;133(5):e1121-e1127. doi:10.1542/peds.20133291.

[32] Kline KA, Lewis AL. Gram-positive uropathogens, polymicrobial urinary tract infection, and the emerging microbiota of the urinary tract. Microbiol Spectr 2016;4(2):UTI-0012-2012. doi:10.1128/microbiolspec.UTI-0012-2012.

[33] Shen Z, Zhong S, Zhu Y, Shao Y, He W, Zhang C, et al. Li L. Infectious microecology in urinary tract and reproductive system. In: Li L, editor. Infectious Microecology. Advanced Topics in Science and Technology in China. Berlin: Springer; 2014. p. 377-409.

[34] Rudy M, Nowakowska M, Wiechula B, Zientara M, Radosz-Komoniewska $\mathrm{H}$. Antibiotic susceptibility analysis of Enterococcus spp. isolated from urine. Przegl Lek 2004;61(5):473-476.

[35] Tehrani FJ, Nikpour S. Effect of health on knowledge, self-efficacy and health behaviours of women with urinary tract infection. Int J Urol Nurs 2014;8(1):3-14. doi:10.1111/ijun.12026.

[36] Sibi G, Kumari P, Kabungulundabungi N. Antibiotic sensitivity pattern from pregnant women with urinary tract infection in Bangalore, India. Asian Pac J Trop Med 2014;7S1:S116-S120. doi:10.1016/S19957645(14)60216-9.

[37] Bassetti M, De Waele JJ, Eggimann P, Garnacho-Montero J, Kahlmeter G, Menichetti F, et al. Preventive and therapeutic strategies in critically ill patients with highly resistant bacteria. Intensive Care Med 2015;41(5):776-795. doi:10.1007/s00134-015-3719-z.

[38] Feglo PK, Acheampong DO, Gbedema SY. Prevalence and Antibiogram of Klebsiella species recovered from clinical samples at Komfo Anokye Teaching hospital in Ghana. J Clin Res Lett 2010;1(2):4-8.

[39] Acheampong DO, Feglo PK. Empirical treatment of neonatal sepsis by Klebsiella: A case study at Komfo Anokye Teaching Hospital (KATH), Ghana. Eur J Exp Bio 2011;1(1):18-22.

[40] Tu Y, McCalla DR. Effect of activated nitrofurans on DNA. Biochimica et Biophysica Acta 1975;402(2):142-149. doi:10.1016/00052787(75)90032-5.

[41] Deitz WH, Cook TM, Goss WA. Mechanism of action of nalidixic acid on Escherichia coli III. Conditions required for lethality. J Bacteriol 1966;91(2):768-773.

[42] Jain A, Roy I, Gupta MK, Kumar M, Agarwal SK. Prevalence of extended-spectrum beta-lactamase-producing Gram-negative bacteria in septicaemic neonates in a tertiary care hospital. J Med Microbiol 2003;52(5):421-425. doi:10.1099/jmm.0.04966-0. 